Article Published in: Physical Review E, DOI: 10.1103/PhysRevE.103.023101

\title{
Squirt flow in porous media saturated by Maxwell-type non-Newtonian fluids
}

\author{
Santiago G. Solazzi,* Beatriz Quintal, and Klaus Holliger \\ Institute of Earth Sciences, University of Lausanne, Lausanne, Switzerland
}

(Received 11 September 2020; accepted 27 December 2020; published 1 February 2021) 


\begin{abstract}
Mechanical waves, which are commonly employed for the non-invasive characterization of fluidsaturated porous media, tend to induce pore-scale fluid pressure gradients. The corresponding fluid pressure relaxation process is commonly referred to as squirt flow and the associated viscous dissipation can significantly affect the waves' amplitudes and velocities. This, in turn, implies that corresponding measurements contain key information about flow-related properties of the probed medium. In many natural and applied scenarios, pore fluids are effectively non-Newtonian, for which squirt flow processes have, as of yet, not been analysed. In this work, we present a numerical approach to model the attenuation and modulus dispersion of compressional waves due to squirt flow in porous media saturated by Maxwell-type non-Newtonian fluids. In particular, we explore the effective response of a medium comprising an elastic background with interconnected cracks saturated with a Maxwell-type non-Newtonian fluid. Our results show that wave signatures strongly depend on the Deborah number, defined as the relationship between the classic Newtonian squirt flow characteristic frequency and the intrinsic relaxation frequency of the non-Newtonian Maxwell fluid. With larger Deborah numbers, attenuation increases and its maximum is shifted towards higher frequencies. Although the effective plane wave modulus of the probed medium generally increases with increasing Deborah numbers, it may, however, also decrease within a restricted region of the frequency spectrum.
\end{abstract}

\title{
I. INTRODUCTION
}

Mechanical waves are commonly employed for the non-invasive characterization of fluidsaturated biological [1], geological [2], and engineered [3] materials. In this context, the probed media are commonly conceptualized as a solid matrix comprising an interconnected void/pore space, which is occupied by a fluid phase [4]. In general, pore fluids are assumed to be Newtonian, implying that their viscosity $\eta$ is a shear-stress and frequency-independent parameter. However, for a wide range of practical applications and natural scenarios, fluids present an effective non-Newtonian behavior [e.g., 5]. For example, fluids employed in hydraulic fracturing and/or drilling operations in porous geological formations are characterized by comprising large concentrations of polymers, surfactants, and/or colloids, which

*santiago.solazzi@unil.ch 
result in non-Newtonian properties [e.g., 6, 7]. As of yet, there is a lack of comprehension of the characteristic signatures of mechanical waves traveling in porous media saturated by non-Newtonian fluids.

Biot's theory of poroelasticity is arguably the most widely used formulation to study wave propagation in porous media saturated by Newtonian fluids [8,9]. Within the framework of this theory, relative motion of the viscous pore fluid with respect to the pore walls can occur in response of a passing wave which, in turn, experiences amplitude loss and phase velocity dispersion due to viscous energy dissipation. Due to this inherent relation between wave characteristics and fluid flow properties, there is significant interest in understanding the physical mechanisms behind the attenuation and dispersion of mechanical waves, which may provide information about the hydraulic properties of the explored media, such as the permeability [e.g., 10].

There are two main fluid-related dissipation mechanisms that can take place in monosaturated porous media: (i) global flow [e.g., 8, 9] and (ii) squirt flow [e.g., 11]. Global flow takes place when the solid frame is accelerated by a passing wave, thus inducing relative fluid displacements with respect to the pore walls. This mechanism is driven by inertial forces and, in the context of geophysical characterization of consolidated geological formations, tends to become relevant at frequencies that are much higher than those typically employed in seismic exploration [e.g. 12]. On the other hand, squirt flow prevails in porous media with locally contrasting compressibilities, such as, for example, interconnected cracks embedded in an otherwise intact matrix, whose characteristic sizes are much smaller than the prevailing wavelengths (i.e., microscopic-mesoscopic scale). Notably, squirt flow effects prevail at much lower frequencies than those of global flow and, thus, may be an important source of energy dissipation in the seismic and sonic frequency band [e.g., 13, 14]. The above described dissipation mechanisms have been studied extensively for porous media saturated with Newtonian fluids (see Müller et al. [10] for a comprehensive review). However, further research is needed in order to understand how these important dissipation mechanisms are affected by the presence of non-Newtonian pore fluids.

Previous efforts to explore the effects of non-Newtonian fluids on the wave signatures of porous media were mainly focused on global flow. Del Rio et al. [15] studied the effects of an oscillating non-Newtonian fluid on a capillary tube to explore the corresponding effects on the dynamic permeability. For this purpose, the non-Newtonian viscosity behavior was 
modeled using a linearly viscoelastic Maxwell-type model. Tsiklauri and Beresnev [6, 16] connected this model to Biot's poroelasticity theory [9] to study global flow dissipation experienced by rotational and dilatational elastic waves, conceptualizing the pore space as a bundle of capillary tubes. These authors demonstrated that the non-Newtonian behavior of the fluid can significantly affect the wave signatures. More recently, the approach of Tsiklauri and Beresnev [6] was used to study Rayleigh wave signatures [17] and guided waves generated in a fluid-filled borehole [18] in porous media saturated with Maxwell-type non-Newtonian fluids. As opposed to global flow effects, which were addressed in the works mentioned above, the effects of squirt flow in porous media saturated by non-Newtonian fluids do, however, remain largely unexplored.

Here, we study squirt flow effects on compressional wave attenuation and dispersion in porous media saturated by a linearly viscoelastic Maxwell-type non-Newtonian fluid. We provide a procedure to include the effects of such a non-Newtonian Maxwell fluid on the squirt flow modeling approach proposed by Quintal et al. [19], which permits to analyze the associated compressional wave signatures for fluid viscosities with different intrinsic relaxation characteristics. To illustrate these effects, we consider a simple model of a porous medium, whose representative elementary volume (REV) consists of two orthogonal and intersecting cracks saturated with a non-Newtonian linear Maxwell fluid embedded in an elastic impervious background.

\section{THEORY}

In the following, we describe a set of equations that permit us to compute squirt flow effects on the compressional wave signatures of porous media for a known pore space topology. For this, we introduce the method of Quintal et al. [19], which considers that the embedding frame is an elastic solid hosting cracks/pores saturated with a Newtonian fluid. Then, we present a simple procedure to include non-Newtonian behavior in the corresponding formulation. 


\section{A. Governing equations for squirt flow with a Newtonian pore fluid}

Let us consider a porous medium whose matrix is an isotropic elastic linear solid hosting a pore space that is saturated by a viscous and compressible Newtonian fluid. Let us assume that this medium is deformed by a passing compressional wave characterized by presenting smalls strains $\left(\sim 10^{-6}\right)$ and a wavelength that is large compared with the characteristic pore size. Furthermore, we assume that the flow within the pores is such that viscous forces dominate over inertial forces [20]. Note that this latter assumption is fulfilled provided that the prevailing frequencies are much smaller than the so-called Biot's frequency, which is associated with the onset of global flow dissipation. In this context, the linearized and quasi-static coupled Lamé-Navier and Navier-Stokes (LNS) equations can be employed to derive the effective frequency-dependent bulk and shear moduli of the system [19]. The corresponding set of equations, which is detailed below, consists of the conservation of momentum and a generalized constitutive equation.

The conservation of momentum is given by

$$
\nabla \cdot \boldsymbol{\sigma}=0
$$

where $\boldsymbol{\sigma}$ denotes the total stress tensor. As the considered medium comprises both solid and fluid domains, it is possible to discriminate between a solid and a fluid contribution within the total stress tensor [19]

$$
\boldsymbol{\sigma}=\varphi \boldsymbol{\sigma}^{s}+(1-\varphi) \boldsymbol{\sigma}^{f}
$$

where $\varphi$ is a spatially variable parameter, which is equal to 1 and 0 in the solid and fluid domains, respectively. The total stress tensor can be divided into a bulk (volumetric) and a deviatoric (shear) part

$$
\boldsymbol{\sigma}=-p \boldsymbol{I}+\boldsymbol{s}
$$

with $p$ denoting the pressure or hydrostatic stress, $\boldsymbol{I}$ the identity, and $\boldsymbol{s}$ the so-called excess stress tensor. Note that Eq. (3) is valid both for the fluid and solid domains.

On the other hand, the strain tensor for both the solid and the fluid corresponds to $\boldsymbol{\epsilon}=\frac{1}{2}\left(\nabla \boldsymbol{u}+\nabla \boldsymbol{u}^{\mathrm{T}}\right)$, with $\boldsymbol{u}$ denoting the displacement vector and $\mathrm{T}$ the transpose. $\boldsymbol{\epsilon}$ can also be divided into a bulk and a deviatoric part

$$
\boldsymbol{\epsilon}=\frac{\operatorname{tr}[\boldsymbol{\epsilon}]}{3} \boldsymbol{I}+\boldsymbol{\varepsilon}
$$


where $\varepsilon$ is the deviatoric strain.

The matrix and the fluid present an elastic response under bulk deformation and the corresponding constitutive equations are given by

$$
-p=K_{\beta} \operatorname{tr}[\boldsymbol{\epsilon}], \text { with } \beta=s, f,
$$

where $K_{s}$ and $K_{f}$ denote the bulk moduli of the solid and fluid, respectively. However, the stress-strain relationship for shear deformation differs in the solid and fluid domains. For the solid, it is given by

$$
\boldsymbol{s}^{s}=2 \mu_{s} \varepsilon
$$

where $\mu_{s}$ is the shear modulus of the solid matrix. Conversely, the stress-strain relationship for the fluid, in the space-frequency domain, is given by

$$
\boldsymbol{s}^{f}=2 \eta_{0} i \omega \varepsilon
$$

with $\eta_{0}$ the shear viscosity of the Newtonian fluid, $i$ the imaginary unity, and $\omega$ the angular frequency. The generalized constitutive equation is thus given by

$$
\boldsymbol{\sigma}=\varphi\left(2 \mu_{s} \boldsymbol{\varepsilon}+K_{s} \operatorname{tr}[\boldsymbol{\epsilon}] \boldsymbol{I}\right)+(\varphi-1)\left(2 \eta_{0} i \omega \boldsymbol{\varepsilon}+K_{f} \operatorname{tr}[\boldsymbol{\epsilon}] \boldsymbol{I}\right) .
$$

Eqs. (1) and (8) can be used to describe the mechanical response of a porous medium comprising solid and fluid domains. At the boundaries between these domains, complexvalued solid and fluid displacements $\mathbf{u}(\mathbf{x}, \omega)$ are considered to be continuous and are thus naturally coupled. For further details regarding the finite-element procedure used to solve the corresponding equations we refer to the work of Quintal et al. [19]. It is important to remark here that, due to the capacity of the viscous Newtonian fluid to flow within the pores in response to a macroscopic deformation, the effective elastic moduli of the medium are complex-valued and frequency-dependent which, in turn, results in attenuation and modulus dispersion of compressional waves, as further explained in subsection IID.

\section{B. Non-Newtonian Maxwell fluid with shear relaxation}

Experimental evidence shows that several non-Newtonian fluids, such as some surfactant solutions, exhibit the rheological behavior of a linear Maxwell fluid [e.g., 21, 22]. In the 
space-frequency domain, the relationship between the excess stress tensor $s$ and the shear rate $i \omega \varepsilon$ for a linear Maxwell fluid responds to [e.g., 5]

$$
\begin{aligned}
\boldsymbol{s}^{f}+\tau_{m} i \omega \boldsymbol{s}^{f} & =2 \eta_{0} i \omega \boldsymbol{\varepsilon}, \\
\boldsymbol{s}^{f} & =2 \eta_{m} i \omega \boldsymbol{\varepsilon},
\end{aligned}
$$

where $\tau_{m}$ is the relaxation time of the corresponding fluid and $\eta_{m}=\eta_{0} /\left(1+\tau_{m} i \omega\right)$ is the frequency-dependent and complex-valued viscosity. We define $\omega_{m}=2 \pi / \tau_{m}$ as the characteristic angular frequency of the intrinsic relaxation of the fluid and, thus, $\eta_{m}$ responds to

$$
\eta_{m}(\omega)=\frac{1}{\left(1+2 \pi i \frac{\omega}{\omega_{m}}\right)} \eta_{0}
$$

The Newtonian regime (Eq. 7) prevails when the fluid has enough time to relax during a wave cycle, that is,

$$
\begin{aligned}
\lim _{\omega / \omega_{m} \rightarrow 0} \boldsymbol{s}^{f} & =\lim _{\omega / \omega_{m} \rightarrow 0} \frac{2 \eta_{0} i \omega}{\left(1+2 \pi i \omega / \omega_{m}\right)} \varepsilon \\
& =2 \eta_{0} i \omega \varepsilon
\end{aligned}
$$

On the other hand, the elastic regime (Eq. 6) prevails when the angular frequency $\omega$ of the traveling wave is such that $\omega_{m}<<\omega$

$$
\begin{aligned}
\lim _{\omega / \omega_{m} \rightarrow \infty} s^{f} & =\lim _{\omega / \omega_{m} \rightarrow \infty} \frac{2 \eta_{0} i \omega}{\left(1+2 \pi i \omega / \omega_{m}\right)} \varepsilon \\
& =\lim _{\omega / \omega_{m} \rightarrow \infty} \frac{2 \eta_{0} \omega_{m}}{2 \pi\left(\omega_{m} / i 2 \pi \omega+1\right)} \varepsilon \\
& =\frac{2 \eta_{0} \omega_{m}}{2 \pi} \varepsilon \\
& =\frac{2 \eta_{0}}{\tau_{m}} \varepsilon \\
& =2 \mu_{f} \varepsilon
\end{aligned}
$$

with $\mu_{f}=\frac{\eta_{0}}{\tau_{m}}$ denoting the shear modulus of the fluid. Consequently, a direct replacement of $\eta_{0}$ for $\eta_{m}$ in Eq. (8) permits to obtain the constitutive equation for a saturating fluid presenting Maxwell-type non-Newtonian shear behavior.

\section{Deborah number}

Squirt flow occurs in response to a fluid pressure diffusion process whose characteristic time, when the medium is saturated with a Newtonian fluid, can be defined as $\tau_{c}=2 \pi / \omega_{c}$, 
with $\omega_{c}$ being the corresponding Newtonian squirt flow characteristic frequency. When considering a Maxwell-type non-Newtonian pore fluid, the interrelationship between squirt flow and the intrinsic shear relaxation of the fluid is a key aspect determining the effective response of the medium. Following previous works $[6,15,16]$, we define the so-called Deborah number $\chi$ of the system, which is determined here as the ratio between the relaxation time of squirt flow with Newtonian fluids $\tau_{c}$ and the intrinsic relaxation time of the non-Newtonian Maxwell fluid $\tau_{m}$, that is,

$$
\chi=\frac{\tau_{c}}{\tau_{m}}=\frac{\omega_{m}}{\omega_{c}} .
$$

The Deborah number $\chi$ determines the pore fluid flow regime. Beyond a certain critical value $\chi^{*}$, the fluid's intrinsic relaxation occurs faster than fluid pressure diffusion and, thus, the fluid behaves as Newtonian during squirt flow. Conversely, for $\chi<\chi^{*}$ the fluid exhibits non-Newtonian viscoelastic behavior during the fluid pressure diffusion process. In this context, it is important to note that Eq. (11) can be expressed as a function of the Deborah number

$$
\eta_{m}=\frac{\chi \omega_{c}}{\left(\chi \omega_{c}+2 \pi i \omega\right)} \eta_{0} .
$$

\section{Dispersion and attenuation of compressional waves}

To estimate the compressional wave attenuation and plane wave modulus dispersion, we solve Eqs. (1) and (8) using suitable boundary conditions in a rectangular REV of the porous medium of interest. The boundary conditions can be conceptualized as an oscillatory relaxation test, which emulates the effects of a vertically traveling compressional wavefield (Figs. 1a and 1b). Recall that we are under the assumption that the prevailing wavelengths $\lambda$ are much larger than the REV side-length $L(\lambda>>L)$. The corresponding test consists in applying a harmonic downward-oriented displacement homogeneously at the upper boundary of the REV. The displacements in the vertical and horizontal directions at the bottom and along the lateral boundaries of the model, respectively, are set to zero (Fig. 1c) [e.g 23, 24].

The upscaled elastic properties of the porous medium saturated with a mobile fluid phase are complex-valued and frequency dependent and, thus, the medium can be regarded as an effective homogeneous viscoelastic solid. Consequently, we can calculate the effective attenuation and dispersion using volume averages of the frequency-dependent stress and strain fields [e.g., 23, 25]. In this context, the complex-valued and frequency-dependent 
plane wave modulus $H$, associated with a compressional wave propagating in the vertical direction $\mathbf{x}_{\mathbf{3}}$, can be approximated by

$$
H(\omega)=\frac{\left\langle\sigma_{33}(\omega)\right\rangle}{\left\langle\epsilon_{33}(\omega)\right\rangle},
$$

where $\langle\cdot\rangle$ denotes the volume average of the corresponding parameters. The attenuation experienced by the wave in such a medium, expressed as the inverse of the quality factor, is given by [e.g., 26]

$$
\frac{1}{Q_{p}(\omega)}=\frac{\Im\{H(\omega)\}}{\Re\{H(\omega)\}},
$$

where $\Im$ and $\Re$ denote the real and imaginary parts, respectively. This approach to compute attenuation and modulus dispersion of compressional waves due to squirt flow has previously been validated and verified [e.g., 19, 24, 27].

\section{RESULTS}

\section{A. Squirt flow effects in a cracked medium}

Following Quintal et al. [19], we consider the scenario of a 2D medium whose REV is a square of side-length $L$ comprising two interconnected orthogonal cracks embedded in an elastic homogeneous background (Fig. 1). The cracks constitute the pore space and are characterized by a length $l_{f}$ and aperture $h_{f}$, such that the aspect ratio is given by $\alpha=h_{f} / l_{f}$ [19]. In the following, we take $\alpha=3.6 \times 10^{-3}$. The side-length of the REV is such that the porosity of the system is $\phi=2 h_{f} l_{f} / L^{2}=0.35 \%$. As long as the geometrical configuration, $\alpha$, and $\phi$ are maintained, and the underlying assumptions are valid, the physical process is completely scalable.

When a compressional wave propagates vertically through the cracked medium (Fig. 1a), it compresses the horizontal cracks, thus increasing the fluid pressure within them, while leaving the fluid pressure in vertical cracks essentially unperturbed. The thus induced fluid pressure gradients arising between the horizontal and the vertical cracks relax through viscous fluid flow. For sufficiently small frequencies, the medium is in a relaxed state, that is, the pressure gradients have time to relax in a half-wave-cycle. In this regime, viscous dissipation is virtually null and the medium presents its lowest stiffness. Conversely, for sufficiently high frequencies, fluid pressure does not have enough time to equilibrate in a 


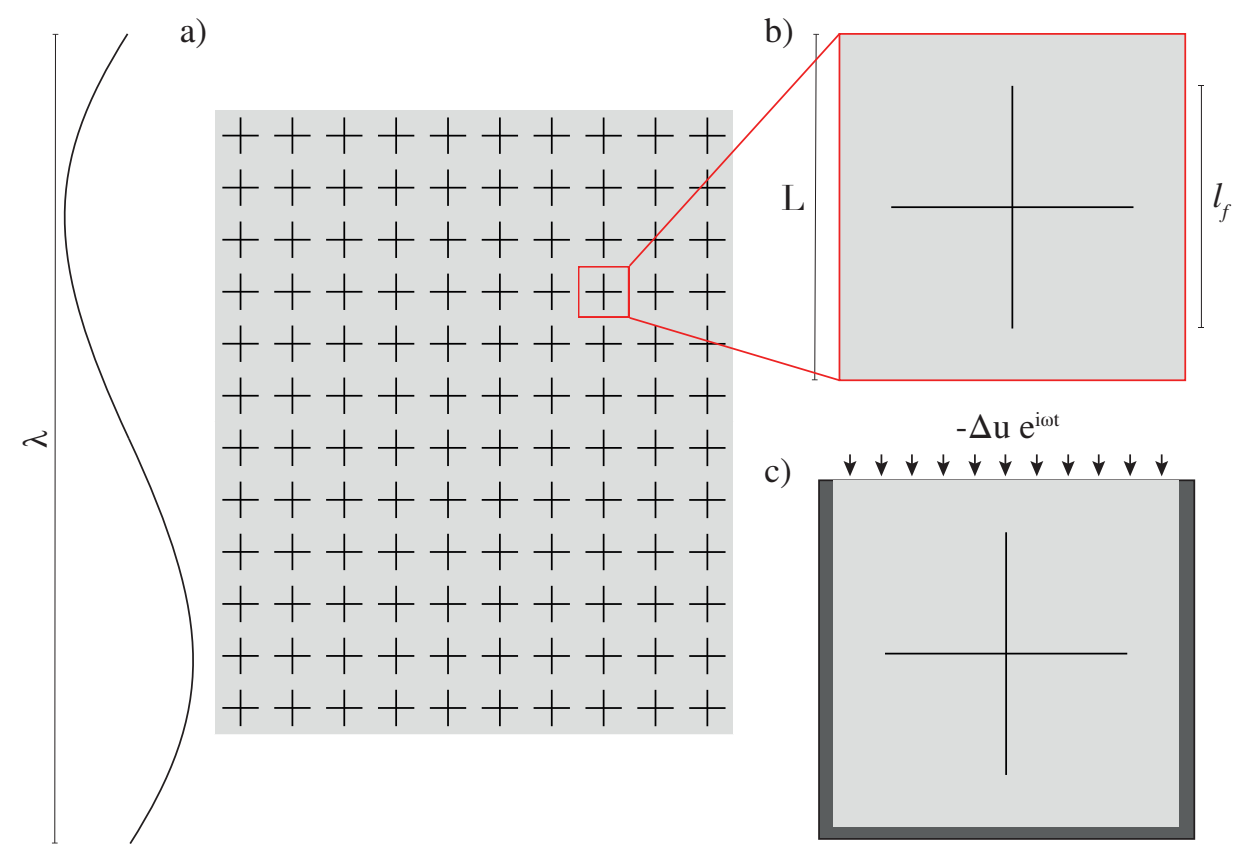

FIG. 1. Schematic illustration of: (a) the probed porous medium; (b) an REV of such medium, which contains a set of interconnected orthogonal cracks; and (c) the oscillatory relaxation test employed to obtain the frequency-dependent overall stress and strain of the medium. Note that the side-length $L$ of the REV is considered to be much smaller than the prevailing wavelengths $\lambda$.

half-wave-cycle and, thus, the medium presents its highest stiffness and viscous flow and dissipation are negligible. Interestingly, for intermediate frequencies, significant fluid flow occurs and, thus, traveling waves can be largely affected by squirt flow .

We study the compressional wave attenuation by analyzing the inverse quality factor $Q_{p}^{-1}$ for different Deborah numbers $\chi$ (Fig. 2). Note that, in Fig. 2, the values of the quality factor are normalized with respect to the maximum attenuation associated with the Newtonian scenario, that is,

$$
\bar{Q}_{p}(\omega, \chi)=\frac{Q_{p}(\omega, \chi)}{Q_{p}\left(\omega_{c}, \infty\right)} .
$$

This characteristic, combined with a normalized frequency $\omega / \omega_{c}$, renders the results independent of $K_{s}, K_{f}$, and $\eta_{0}$. We observe that when $\chi$ is sufficiently high $\left(\chi \geq 10^{4}\right)$, the attenuation curve follows that of a squirt flow process in the presence of a Newtonian fluid. However, with decreasing $\chi$-values, the non-Newtonian behavior of the fluid becomes more pronounced. As a result, attenuation decreases and the frequency associated with the peak attenuation $\omega_{\max }$ is shifted towards lower values (Fig. 2). Interestingly, when the non- 


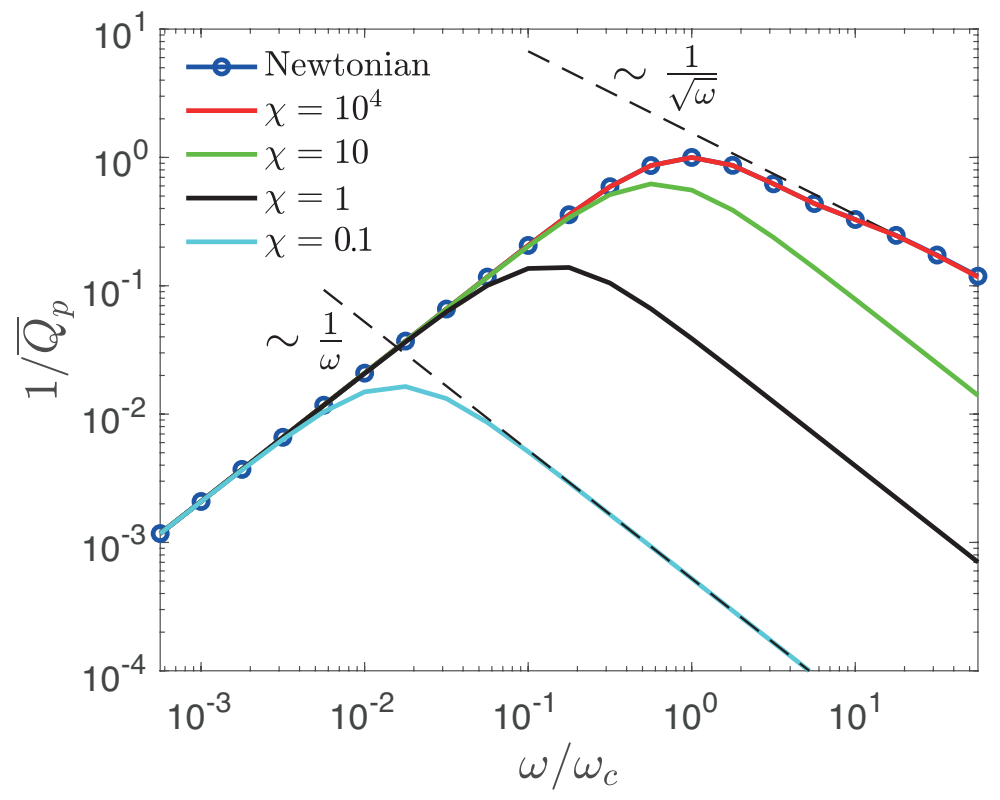

FIG. 2. Normalized inverse quality factor $\bar{Q}_{p}^{-1}$ as a function of the normalized frequency $\omega / \omega_{c}$ for the model shown in Fig. 1. We illustrate the results considering a Newtonian pore fluid (blue line with circles) and Maxwell-type non-Newtonian fluids for different Deborah numbers (colored solid lines). Dashed black lines denote the high-frequency asymptotic behavior of the Newtonian and non-Newtonian fluids.

Newtonian behavior of the fluid becomes dominant, the high-frequency asymptotic behavior of the attenuation changes from $\sim 1 / \sqrt{\omega}$, which is the typical asymptote of squirt flow for Newtonian fluids [13], to $\sim 1 / \omega$. This is an interesting characteristic that may permit, in well constrained scenarios, to discern whether the saturating fluid presents a Newtonian or non-Newtonian characteristics from wave arrival observations.

As a consequence of the squirt flow process, the real part of the plane wave modulus $H$ increases, evidencing a stiffening effect with increasing frequencies. This characteristic is illustrated in Fig. 3, which shows the real part of the plane wave modulus normalized respect to its low-frequency Newtonian counterpart

$$
\Re\{\bar{H}(\omega, \chi)\}=\frac{\Re\{H(\omega, \chi)\}}{\Re\{H(0, \infty)\}} .
$$

We note that dispersion is more pronounced for high values of the Deborah number $\chi$, emulating the Newtonian behavior (Fig. 3). With decreasing values of $\chi$, the dispersion decreases, as does the inflection of the dispersion curve moves towards lower frequencies. This is expected, as the inflection in the dispersion curve is associated with the frequency 


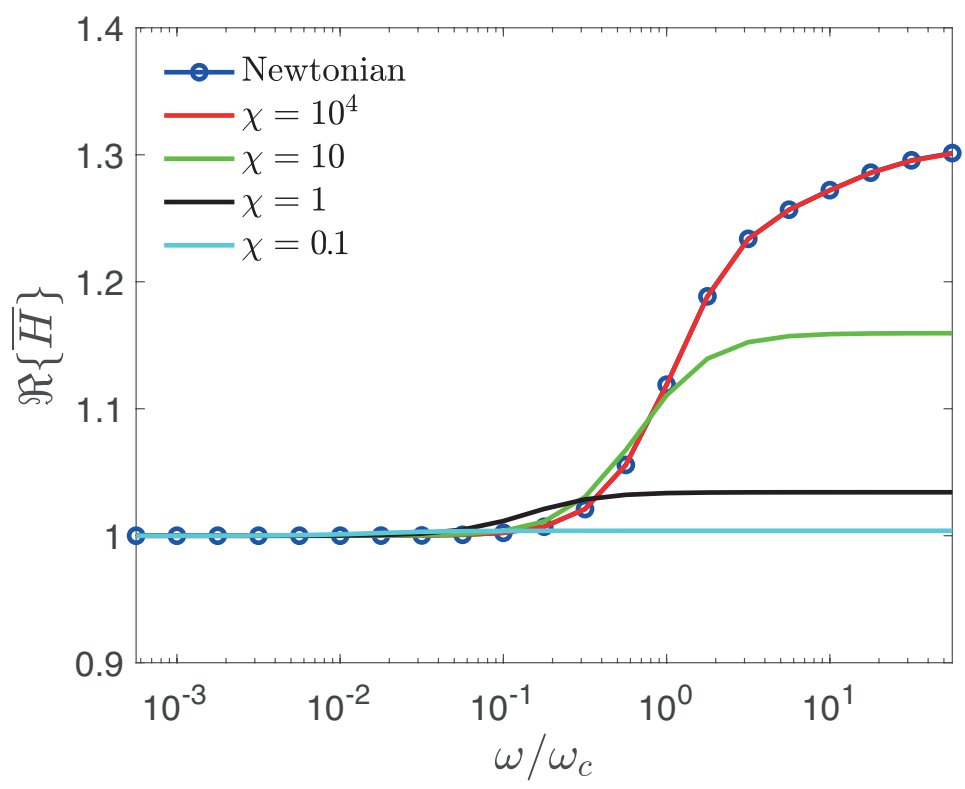

FIG. 3. Real part of the normalized effective bulk modulus $\Re\{\bar{H}\}$ as a function of the normalized frequency $\omega / \omega_{c}$ for the model illustrated in Fig. 1. We illustrate the results considering a Newtonian pore fluid (blue line with circles) and non-Newtonian fluids with different Deborah numbers (colored solid lines).

corresponding to the attenuation peak $\omega_{\max }$, which also moves towards lower frequencies for decreasing values of $\chi$ (Fig. 2). It is interesting to observe that, even though $\Re\{\bar{H}\}$ generally increases with $\chi$, this is not true across the entire frequency band. In a narrow frequency range near the inflection of the dispersion curve, smaller $\chi$-values are associated with slightly higher $\Re\{H\}$ values, which, in turn, could result in higher compressional wave velocities.

\section{B. Deborah number and peak frequency}

The frequency associated with the maximum attenuation $\omega_{\max }$ changes with the Deborah number $\chi$ (Fig. 2). In the classic squirt flow mechanism for porous/cracked media saturated with Newtonian fluids, the peak frequency, which, in this case, is given by $\omega_{c}$, fulfills [e.g., $28,29]$

$$
\omega_{c} \propto \frac{K_{s}}{\eta_{0}} \alpha^{3} .
$$


This implies that the peak frequency depends on the geometrical characteristics of the fluidfilled pore space. In the particular case studied here, $\omega_{\max }$ depends on the aspect ratio of the cracks $\alpha$. Further knowledge regarding the relationship between $\omega_{\max }$ and $\chi$ may permit to discern variations in the compressional wave signatures related to the presence of non-Newtonian fluids from those associated with changes the crack aspect ratio.

The relationship between $\omega_{\max }$ and $\chi$ is displayed in Fig. 4 (black circles). This relationship is retrieved by computing the attenuation curves as functions of frequency for several $\chi$ values, including those illustrated in Fig. 2, and selecting the maxima of the corresponding curves. This is done following the approach described in Section II. We find that the relationship between $\omega_{\max }$ and $\chi$ can be approximated by the following empirical formula

$$
\frac{\omega_{\max }}{\omega_{c}} \simeq\left(1+\frac{2 \pi}{\chi}\right)^{-1}
$$

which is illustrated in Fig. 4 using red dashed lines.

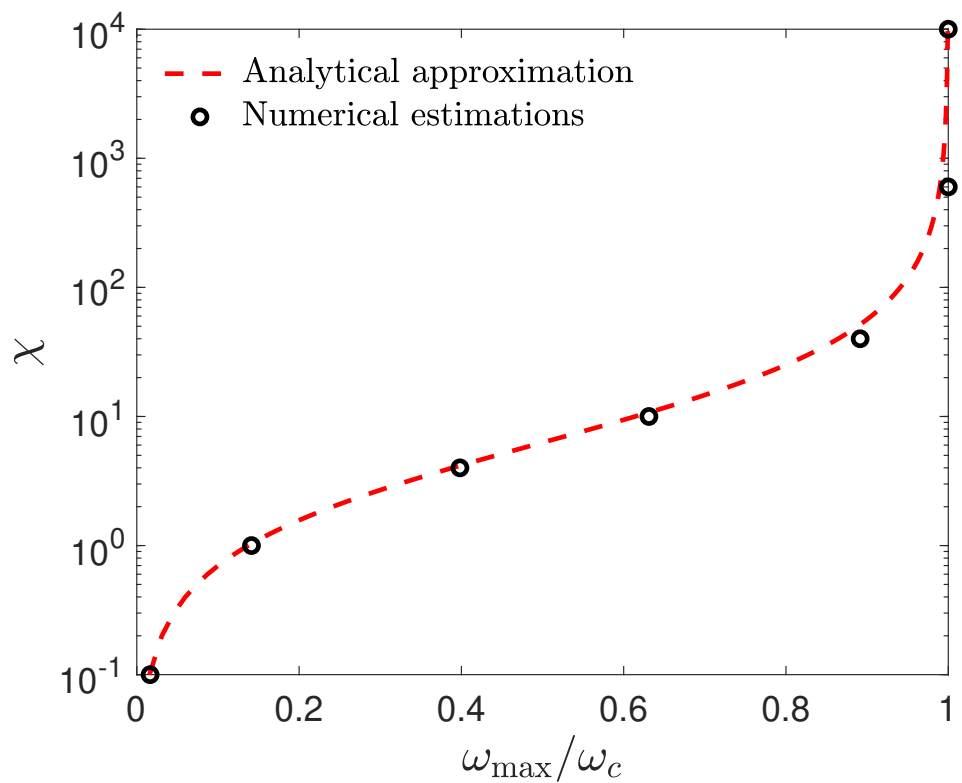

FIG. 4. Relationship between the normalized maximum frequency $\omega_{\max } / \omega_{c}$ and the Deborah number $\chi$. We compare the values obtained from the numerical estimations (black circles) and the analytical approximation given by Eq. (27) (red dashed line). 


\section{DISCUSSION}

We have explored squirt flow on attenuation and plane wave modulus dispersion of mechanical waves in presence of Maxwell-type non-Newtonian pore fluids using an approach based on continuum mechanics. To this end, we consider small strains, wavelengths which are much larger than the pore scale, and Poiseuille-type flow. These assumptions have been proven to be valid and pertinent to model squirt flow effects in a great variety of scenarios in earth sciences, where cracks/pores range from $\mu \mathrm{m}$ - to mm-scale. However, some of the assumptions may be inadequate for very small pores (i.e., molecule scale). For instance, in nanometer-scale pores, the molecules of certain fluids can present a non-zero tangential velocity at the solid-fluid interface, thus rendering the no-slip condition invalid [e.g., 30].

Several aspects of the mechanical response of rocks saturated by linear Maxwell-type fluids were analyzed by Tsiklauri and Beresnev $[6,16]$. These authors explored the effects of non-Newtonian viscoelastic fluids on wave attenuation and dispersion due to global flow, that is, when inertial forces prevail over viscous forces. The results of these studies are therefore complemented by our work, which focuses on corresponding squirt flow effects. It is important to remark here that our analysis is based on viscoelastic Maxwell fluids with stress relaxation, which are a pertinent representation of some polymeric liquids [e.g., 5]. However, an extension of the corresponding results to non-Newtonian fluids in general (i.e., colloidal suspensions and/or polymeric fluids) is not straightforward. In this sense, alternative non-Newtonian fluid behaviors, such as, shear thinning, shear thickening, and viscoplastic fluids, would require different modelling approaches and upscaling techniques.

\section{CONCLUSIONS}

We have presented an approach that permits to include non-Newtonian behavior of Maxwell-type fluids into the viscous dissipation mechanism associated with squirt flow. Our results show that the resulting attenuation and modulus dispersion strongly depend on the Deborah number, which is a measure of the relaxation time of the Maxwell-type fluids with regard to that of the the Newtonian squirt flow process. For increasing Deborah numbers, that is, for non-Newtonian fluids with relatively fast Maxwell-type relaxation, the compressional wave attenuation tends to increase and its peak frequency moves towards 
higher frequencies. Interestingly, the non-Newtonian behavior of pore fluids affects the highfrequency asymptote of the attenuation curves, which becomes inversely proportional to the angular frequency. This characteristic is important as, under well-controlled conditions, it might help to discern whether the medium is effectively saturated by a non-Newtonian fluid based on wave arrival observations. We also note that the plane wave modulus of the medium increases with the Deborah number. However, the plane wave modulus may also decrease within a restricted frequency range around the inflection of the dispersion curve for increasing Deborah numbers. Within this frequency range, the velocity of the compressional waves could therefore increase in response to the displacement of a Newtonian pore fluid by a non-Newtonian phase. Finally, we show that a non-linear relationship exists between the Deborah number and the frequency of the maximum attenuation. The results of this study fundamentally improve our understanding of the squirt flow attenuation mechanism in porous media saturated by non-Newtonian fluids and, thus, provide the basis for advancing corresponding detection and interpretation techniques for a wide range of applications.

\section{ACKNOWLEDGMENTS}

This study was completed within the Swiss Competence Center for Energy ResearchSupply of Electricity with support of Innosuisse.

[1] S. C. Cowin, J. Biomech. 32, 217 (1999).

[2] E. Detournay and A. H.-D. Cheng, in Analysis and design methods (Elsevier, 1993) pp. 113171.

[3] P. Chiarelli, A. Lanatà, M. Carbone, and C. Domenici, J. Acoust. Soc. Am. 127, 1197 (2010).

[4] J. Bear, Dynamics of Fluids in Porous Media (Elsevier, N. Y., 1972).

[5] R. Bird, R. Armstrong, and O. Hassager, (1987).

[6] D. Tsiklauri and I. Beresnev, Phys. Rev. E 63, 046304 (2001).

[7] T. Friedel, in SPE Gas Tech. Symp. (Society of Petroleum Engineers, 2006).

[8] M. A. Biot, J. Acoust. Soc. Am. 28, 168 (1956).

[9] M. A. Biot, J. Acoust. Soc. Am. 28, 179 (1956). 
[10] T. M. Müller, B. Gurevich, and M. Lebedev, Geophysics 75, 147 (2010).

[11] J. Dvorkin, G. Mavko, and A. Nur, Geophysics 60, 97 (1995).

[12] S. R. Pride, in Hydrogeophysics, edited by Y. Rubin and S. Hubbard (Springer, 2005) Chap. 9, pp. 253-290.

[13] B. Gurevich, D. Makarynska, O. B. de Paula, and M. Pervukhina, Geophysics 75, N109 (2010).

[14] M. Chapman, Geophys. Prospect. 51, 369 (2003).

[15] J. A. Del Rio, M. L. De Haro, and S. Whitaker, Phys. Rev. E 58, 6323 (1998).

[16] D. Tsiklauri and I. Beresnev, Transp. Porous Med. 53, 39 (2003).

[17] M. Markov, Acoust. Phys. 52, 429 (2006).

[18] C. Zhi-Wen, L. Jin-Xia, Y. Gui-Jin, and W. Ke-Xie, Chin. Phys. B 19, 084301 (2010).

[19] B. Quintal, E. Caspari, K. Holliger, and H. Steeb, Geophys. Prospect. 67, 2196 (2019).

[20] D. L. Johnson, J. Koplik, and R. Dashen, J. Fluid Mech. 176, 379 (1987).

[21] J. Castrejón-Pita, J. Del Río, A. Castrejón-Pita, and G. Huelsz, Phys. Rev. E 68, 046301 (2003).

[22] M. Torralba, J. Castrejón-Pita, A. Castrejón-Pita, G. Huelsz, J. Del Río, and J. Ortín, Phys. Rev. E 72, 016308 (2005).

[23] J. G. Rubino, C. L. Ravazzoli, and J. E. Santos, Geophysics 74, N1 (2009).

[24] B. Quintal, J. G. Rubino, E. Caspari, and K. Holliger, Geophysics 81, D335 (2016).

[25] S. G. Solazzi, J. G. Rubino, T. M. Müller, M. Milani, L. Guarracino, and K. Holliger, Geophys. J. Int. 207(2), 823 (2016).

[26] R. J. O'Connell and B. Budiansky, Geophys. Res. Lett. 5, 5 (1978).

[27] Y. Alkhimenkov, E. Caspari, B. Gurevich, N. D. Barbosa, S. Glubokovskikh, J. Hunziker, and B. Quintal, Geophysics 85, MR129 (2020).

[28] R. J. O'Connell and B. Budiansky, J. Geophys. Res. 82, 5719 (1977).

[29] S. Lissa, N. D. Barbosa, E. Caspari, Y. Alkhimenkov, and B. Quintal, JGR: Solid Earth 125, e2019JB019235 (2020).

[30] D. Ortiz-Young, H.-C. Chiu, S. Kim, K. Voïtchovsky, and E. Riedo, Nat. Commun. 4, 1 (2013). 